\title{
Prediction of Deepwater FPSO responses using different numerical analysis methods
}

\author{
Matthew Guan ${ }^{1, *}$, Montasir Osman ${ }^{1}$, and Cheng Yee $\mathrm{Ng}^{1}$ \\ ${ }^{1}$ Civil and Environmental Engineering Department, Universiti Teknologi PETRONAS, 32610 Bandar \\ Seri Iskandar, Perak, Malaysia
}

\begin{abstract}
The limitations of existing wave basins present a significant challenge when modelling offshore deepwater systems, particularly due to the basin's relatively shallow depth. Numerical simulation thus becomes valuable in predicting its behaviour during operation at sea. The coupled dynamic analysis is preferred over the traditional quasi-static method, as the former enables the inclusion of damping and added mass properties of the complete mooring line system, which becomes increasingly prominent at greater water depths. This paper investigates the motions and mooring line tensions of a turret moored Floating Production Storage Offloading (FPSO) platform using three numerical models, i.e. a dynamic system, quasi-static system and linear spring system subjected to unidirectional random wave condition. Analysis is carried out using a commercial software AQWA. The first two numerical models utilise a complete system of the same setup and configuration, while the linear spring system substitutes the mooring lines with equivalent linear springs and attempts to match the total mooring line restoring forces with that of the coupled dynamic analysis. The study demonstrates the significance of coupled dynamic analysis on the responses of an FPSO in deepwater. The numerical model of the FPSO is validated against the results of a published work.
\end{abstract}

\section{Introduction}

Floating platforms play an important role in the offshore oil and gas industry. Vessels such as the ship-shaped FPSOs are commonly used in the production of oil and gas in offshore deepwater regions. The system is held in place by mooring lines anchored to the seabed during operation. Due to the cost and complexity of manufacturing a floating platform, extensive design and model testing is vital in ensuring the performance and safety of the prototype before installation and operation. As exploration and production progresses into deeper waters, experimental and analytical studies become increasingly crucial to predict the responses and mooring line/riser tensions in these remote and harsh environments.

Physical modelling is traditionally used in predicting platform prototype behaviour and testing new concepts. Ideally a complete system should be tested in order to account for the dynamic effects induced by the mooring lines. Tests with scales up to 1:150 showed the

\footnotetext{
* Corresponding author: matthewgzh@gmail.com
} 
possibility of achieving good results [1]; however, acute control of environment parameters is necessary for experimental testing on smaller scales, as the model becomes highly sensitive to environment input.

Truncated systems are used for cases where complete system tests are unfeasible. This method can further be divided into passive systems and active systems. For passive systems, the mooring lines are commonly modelled using some mechanical means (e.g. springs) at the cut-off section. This approach ignores the dynamic effects of the mooring line however, and has been proven to yield conservative results. An experiment was conducted by comparing two truncated semisubmersible models with that of a full depth system [2]. The three were designed to have matching static offset curves. It was discovered that the dynamic tensions were underestimated in the truncated systems compared to the full length mooring.

To account for the truncated portion, Stansberg et al. [1] combined physical modeling with numerical simulations to assess the viability of passive hybrid model testing for floating structures [1]. The system was designed to achieve a good reproduction of the total restoring force stiffness and quasi-static offset characteristics of a full depth system. A time domain coupled analysis program RIFLEX-C was applied to simulate the dynamic portion of the system. The results obtained after numerical extension to full depth were promising. Currently hybrid model testing is recommended and extensively used in offshore industry.

In an active truncated system, computer controlled devices at the cutoff portion of the mooring/riser system simulate the effects at the truncated portion in real time. One research project initiated at MARINTEK reexamines the feasibility of a proposed active system HydeMoor [3]. The study used a single mooring line to demonstrate the principles of HydeMoor, in which the effect of the lower portion below the cut-off point was simulated using a numerical modeling run on a computer, and applied to the upper physically modeled portion of the line at the truncation point by means of an actuator. The results showed the possibility of achieving good synchronization of truncation points in the system.

In recent years, numerical simulation has become an attractive option for model testing. These may function as an alternative when inadequate facilities are available for physical testing. Additionally, numerical simulations may reduce the amount of cost as well as time in manufacturing a physical system. Technip has recently modified their design process via the development of a numerical wave basin [4]. This was built on the features provided by the commercial software STAR-CCM+, and coupled with their in-house program to improve certain functionalities that was yet fully developed. Validation of the numerical basin against experimental results showed good agreement after calibration.

In this paper, the motions and line tensions of a turret moored FPSO are studied by applying a coupled dynamic analysis simulation. The results are first validated against a published numerical simulation and its corresponding experiment conducted by Kim et al. [5]. The same model is then later compared with two statically equivalent numerical simulations, a quasi-static analysis and a linear spring system.

\section{Numerical Modelling}

The general concept of the three systems are described, as well as the setup for the numerical model AQWA used in this study. 


\subsection{Dynamic system}

In analyzing the mooring lines of the dynamic system, the effects of mass, drag and inline elastic tension of the line are considered. The mooring line is discretized along the length of the entire line into a finite number of elements subjected to external environmental forces. Combining the cable dynamics with the hull responses, the following equation of motion is solved through coupled time domain analysis:

$$
\left[M+M_{a}(\infty)\right] \ddot{u}_{p}+\int_{0}^{\infty} R(t-\tau) \dot{u}_{p} \mathrm{~d} \tau+K_{H} u_{p}=F_{D}+F^{(1)}+F^{(2)}+F_{p}+F_{w}+F_{c}+F_{W D}
$$

where $M$ and $M_{a}$ are the mass and added mass respectively, $R=$ retardation function, $K_{H}=$ hydrostatic restoring coefficient, $F_{D}=$ drag force on the hull, $F^{(1)}$ and $F^{(2)}=1^{\text {st }}$ and $2^{\text {nd }}$ wave load on the hull respectively, $F_{p}=$ transmitted force from the mooring lines to the hull, $F_{w}=$ wind loading, $F_{c}=$ current loading on hull, and $F_{W D}=$ wave drift damping. The process is computed iteratively for each time interval.

\subsection{Quasi-static system}

In the quasi-static system, the multiple segments in each line is defined by its stiffness, length, mass per unit length and equivalent cross-sectional area. The drag and inertia components on the line are neglected, and the environmental forces acting on the system are considered as static forces. The system's initial position is first obtained, and the static analysis of the complete system is carried out. The simulation is solved iteratively at each time interval, similar to the dynamic system.

\subsection{Linear spring system}

The simplest of the three models, the mooring line is modelled as a massless linear elastic cable. Taking $k=$ stiffness, $L=$ stretched line length, and $L_{0}=$ unstretched line length, the line tension is given as:

$$
T=k\left(L-L_{0}\right)
$$

This theory allows tension but not compression forces in the line, hence $T=0$ if $L<L_{0}$.

\subsection{FPSO model and environmental conditions}

The AQWA model attempts to reproduce as close as possible the same model as given in [5], in which the main particulars of the FPSO, its mooring systems as well as its corresponding hydrodynamic coefficients are also referred to. The arrangement of the mooring lines is shown in Fig. 1. All 12 lines are modelled in AQWA.

Fig. 1. Mooring arrangement.

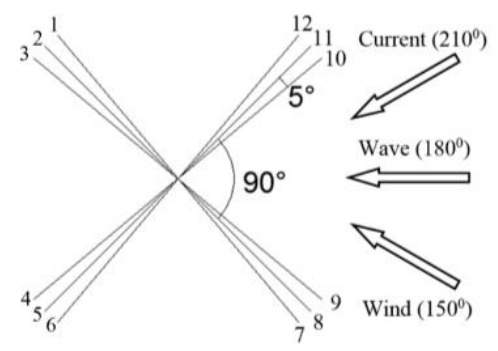


In the published work [5], computation of the second order radiation/diffraction for the hull was carried out using Newman's approximation in order to avoid the more computationally expensive QTF method. The former can be reasonably applied when the natural frequency of the floating system is small and the slope of the QTFs near the diagonal is not large. In the case of AQWA, the more accurate full QTF was used for computation.

\section{Results and discussions}

\subsection{Model validation}

A 100-year storm condition at the Gulf of Mexico was simulated, the details of which can be found in [5]. For the wind and current force considerations, reference was made to OCIMF [6]. The wind and current coefficients were obtained via linear interpolation in order to obtain the values at $80 \%$ loading condition.

The surge static offset was first carried out by displacing the FPSO at varying distances, and plotting the total force in the surge direction, as well as the tensions in the most and least loaded line. The AQWA results were compared with the published work in Fig. 2 and 3.

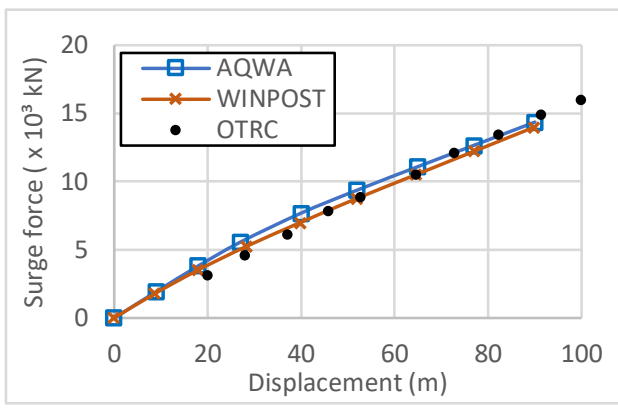

Fig. 2. Static offset results.

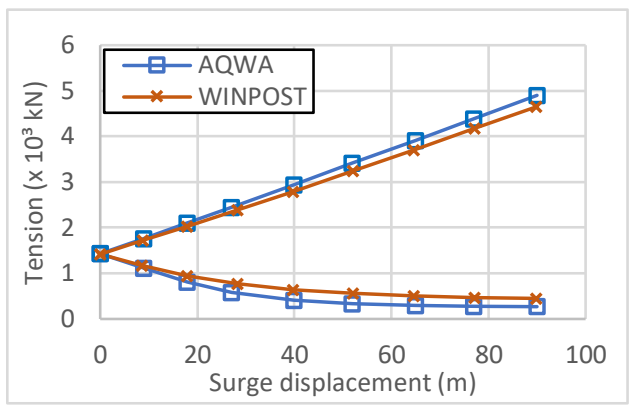

Fig. 3. Tension of most loaded and least loaded line in static offset.

The system's total damping was obtained through free decay tests by displacing the hull at a distance/angle in calm water, and then releasing it to allow it to oscillate freely. The summary comparison is shown in Table 1.

Table 1. Summary of free decay comparison.

\begin{tabular}{|c|c|c|c|c|c|c|}
\hline \multirow{2}{*}{ Motion } & \multicolumn{3}{|c|}{ Period (s) } & \multicolumn{3}{c|}{ Damping ratio (\%) } \\
\cline { 2 - 7 } & OTRC & WINPOST & AQWA & OTRC & WINPOST & AQWA \\
\hline Surge & 206.8 & 204.7 & 192.6 & 3.0 & 4.4 & 12.2 \\
\hline Heave & 10.7 & 10.8 & 11.1 & 6.7 & 11.8 & 12.5 \\
\hline Roll & 12.7 & 12.7 & 12.8 & 3.4 & 0.7 & 0.5 \\
\hline Pitch & 10.5 & 10.8 & 10.1 & 8.0 & 10.5 & 13.5 \\
\hline
\end{tabular}

Fig. 4 to 9 represents the 6 degrees of freedom (DOF) motion responses of the system. The results were first obtained via time domain analysis and later converted to spectral density. It is observed that the surge, sway and yaw are dominated by low frequency (LF) motions, while the heave, roll and pitch are wave frequency (WF) dominated. The surge, heave and pitch show good agreement among the three tests, while the roll spectrum in 
AQWA lie between that obtained from WINPOST and OTRC. The discrepancies with WINPOST may be attributed by a couple of factors such as the minor differences in FPSO model design and the calculation of full QTF matrix in AQWA.

Substantial sway and yaw motions are recorded in the AQWA simulation. This is mainly due to the exclusion of hull viscous effects, which are ignored in the 3D potential diffraction/ radiation theory. Additional hull drag damping was manually input in the WINPOST simulation, which was not carried out in AQWA, in order to match that of the OTRC experiment.

The larger surge, sway and roll are recorded to have an effect on the mooring line tensions (Fig. 10-13). In most cases, the line tensions in AQWA are lower than WINPOST, possibly due to the effect of the exclusion of viscous hull drag. It is also noted that the LF components of tension are significantly higher than the WF components.

Disregarding the magnitudes, it is observed that the three tests display similar trends in the respective spectrums. Overall, the AQWA simulation yields satisfactory results when compared to the published literature.

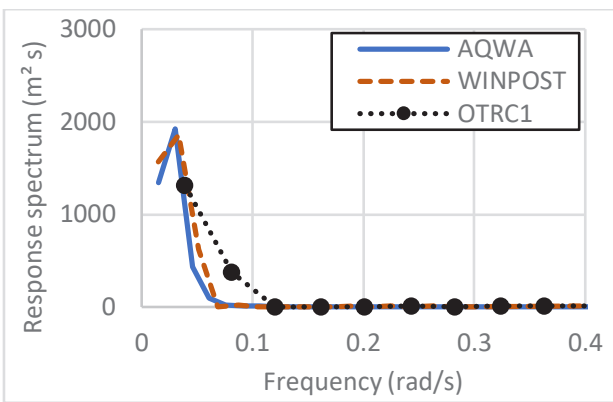

Fig. 4. Surge response.

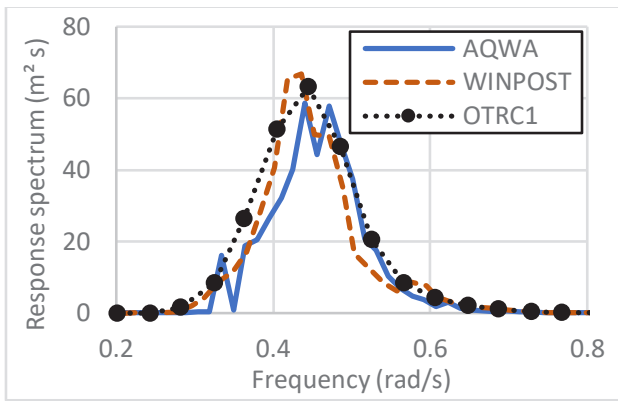

Fig. 6. Heave response.

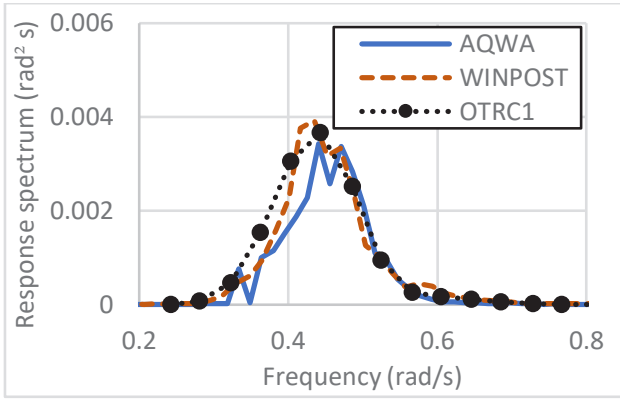

Fig. 8. Pitch response.

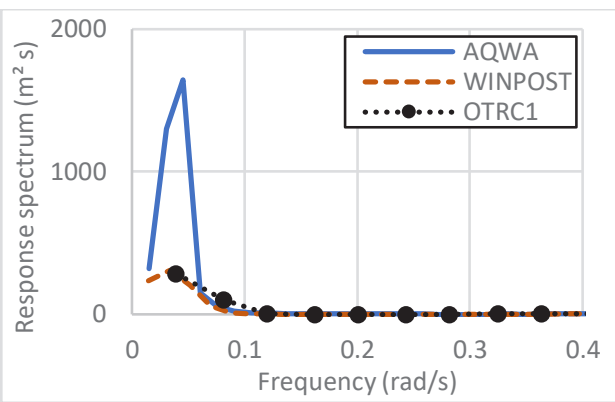

Fig. 5. Sway response.

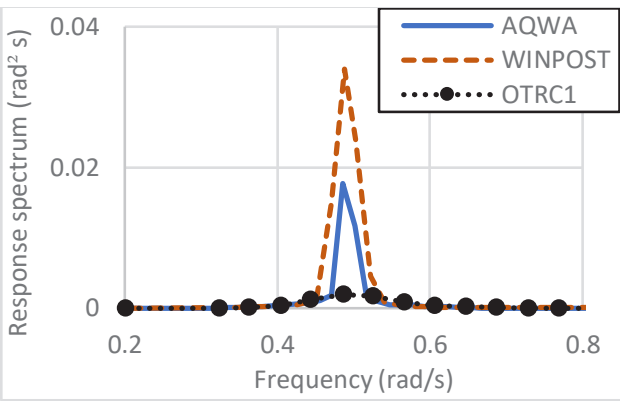

Fig. 7. Roll response.

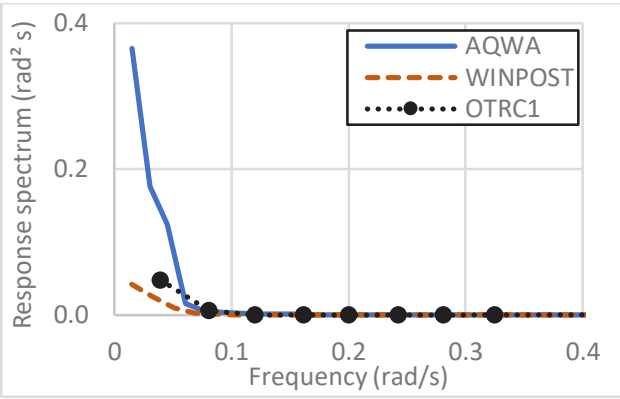

Fig. 9. Yaw response. 


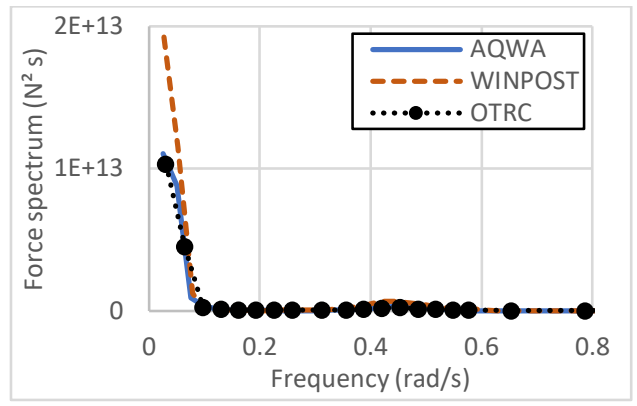

Fig. 10. Combined tensions of lines 1,2 and 3.

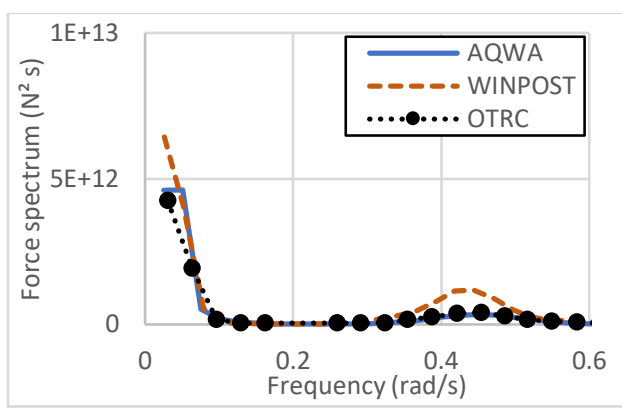

Fig. 12. Combined tensions of lines 7,8 and 9. 12.

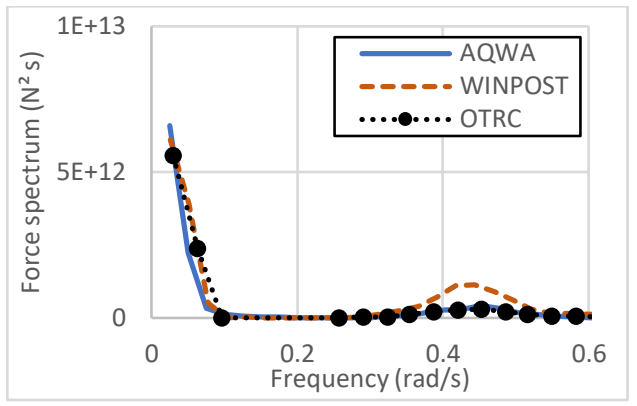

Fig. 11. Combined tensions of lines 4, 5 and 6 .

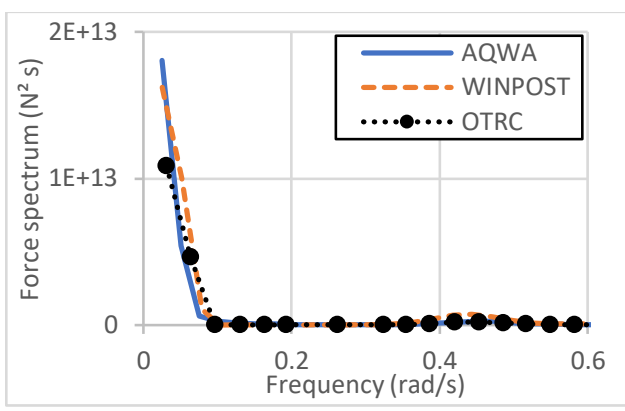

Fig. 13. Combined tensions of lines 10,11 and

\subsection{Dynamic, Quasi-static and Spring Simulation}

Unlike in the validation stage, the analyses simulate only the same wave condition in [5], while disregarding the current and wind effects on the FPSO. The previous AQWA model is renamed as the dynamic system and denoted as D. Two additional simulations were modelled: quasi-static (non-dynamic) system and spring system, denoted as ND and SPR respectively. The hull properties remain the same for the three models, however four equivalent linear springs are used in the SPR system instead of the full 12 catenary lines as with D and ND.

The static offset was first conducted, similar to the validation stage (Fig. 14-15). The results of D and ND were found to be the same, and thus placed in the same line. While the resultant surge force for all three cases matches well for the range of surge motions tested, the combined tension of the major loaded lines 7, 8, and 9 for D and ND are significantly higher than its equivalent spring counterpart of the SPR system. However, the stiffness is greater for the SPR, as can be noted in Figure 15 by the higher rate of tension increment against the displacement. Additionally, the static offset of SPR exhibit a linear increase in surge force as opposed to the non-linear weakening behavior of the D and ND system.

The free decay tests were omitted, as matching the damping of the three systems was not considered in this study. 


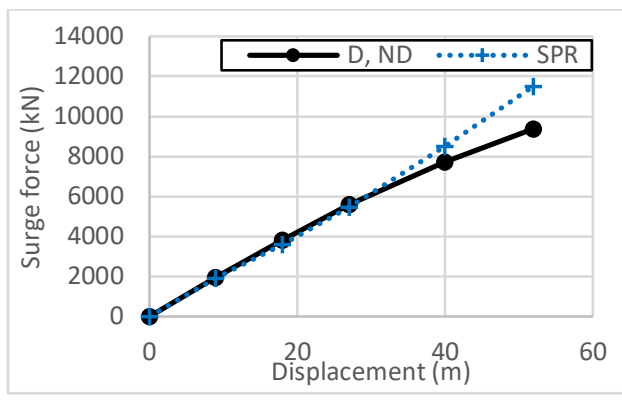

Fig. 14. Surge static offset.

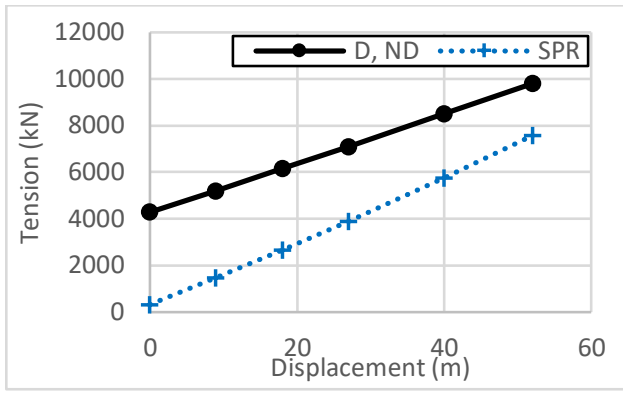

Fig. 15. Combined tension of lines 7,8 and 9.

The station keeping results are shown in Figures 16 to 21 . The sway and yaw in unidirectional waves are expected to be insignificant and hence not included, while the combined tensions $6,7,8$ and $10,11,12$ is expected to mirror $1,2,3$ and 7, 8, 9 respectively. It is observed that the WF heave, pitch and roll responses agree well among the three tests, with the exception of the roll for SPR, which overpredicts the motion. However, both ND and SPR overpredict the surge motion. The surge in D is significantly lower as the damping induced from the mooring lines significantly reduce its low frequency motions. For the surge, ND and SPR are almost similar in energy content. This can be expected, as the SPR system can be considered as a type of quasi-static system, but without line mass. Good agreement for ND and SPR is also observed for the combined tensions of the major loaded lines 7, 8, and 9, as surge is the primary contributor to mooring line tension. The combined tensions spectrum of the minor loaded lines 1, 2, and 3 of SPR is smaller than ND due to its shorter line length.

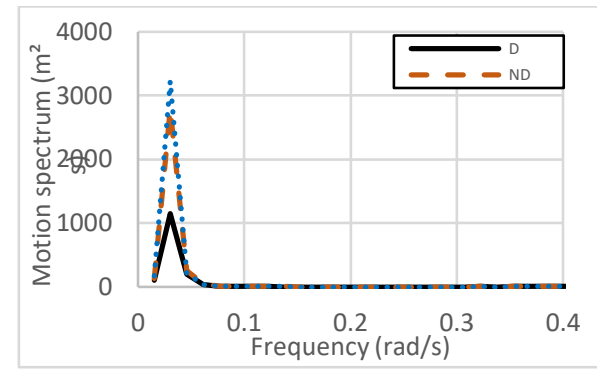

Fig. 16. Surge response.

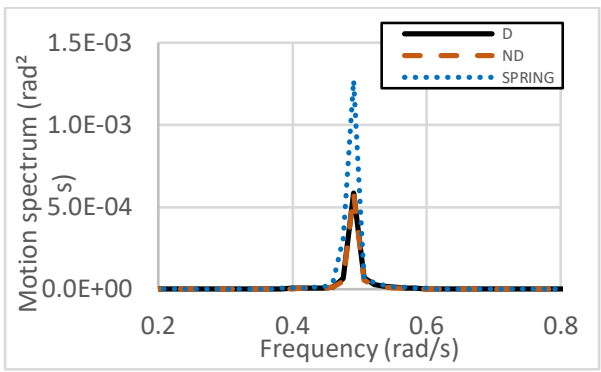

Fig. 18. Roll response.

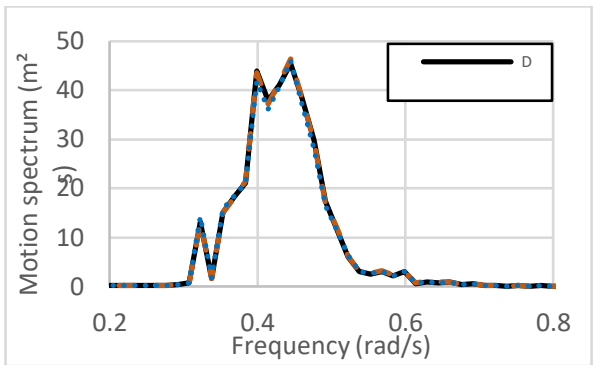

Fig. 17. Heave response.

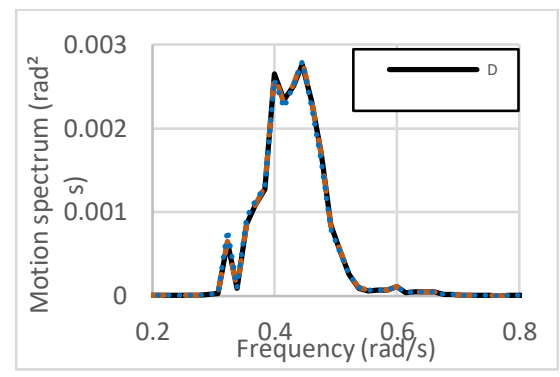

Fig. 19. Pitch response. 


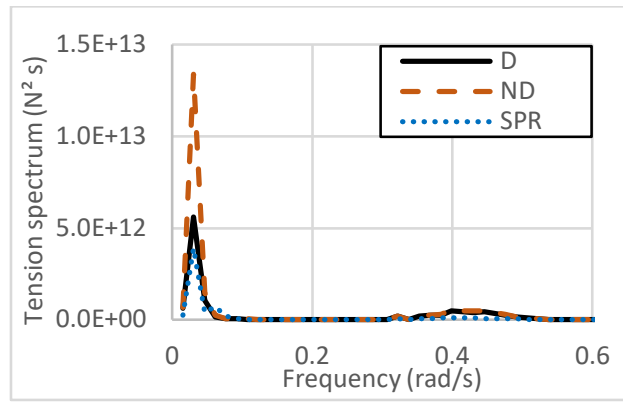

Fig. 20. Combined tensions of lines 1,2 and 3.

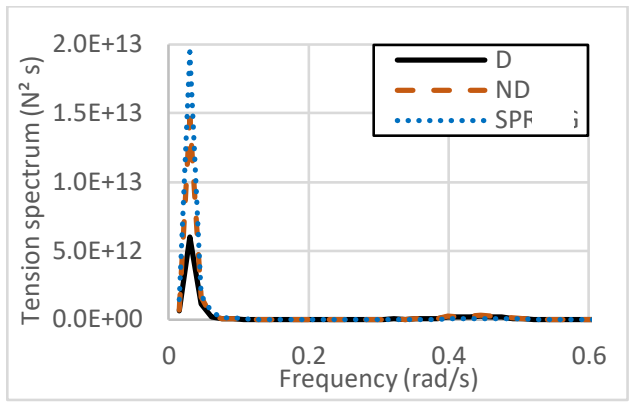

Fig. 21. Combined tensions of lines 7, 8 and 9 .

\section{Conclusions}

The motion responses and line tensions of a turret moored FPSO are obtained using a coupled dynamic analysis program. The results were validated against a published physical test and numerical analysis. The same model was then compared with an equivalent quasistatic system and a linear spring system, under unidirectional wave condition. The heave, roll and pitch are generally in agreement among the three cases, while the surge is overpredicted in the quasi-static and spring system. Consequently, the major loaded line tensions of the two cases are larger than the dynamic system, while the minor loaded line tension for SPR is lower than ND due to its shorter line length. The findings will be used in further research to develop an economical method to improve physical model test results of motions and line tensions in deepwater.

The authors would like to thank the Ministry of Higher Education Malaysia (MOHE), to which the project was funded under the FRGS grant.

\section{References}

1. C.T. Stansberg, O. Øritsland, Marintek, G. Kleiven, Norsk Hydro, Offshore Technology Conference, 12087 (2000)

2. X. Chen, J. Zhang, P. Johnson, M. Irani, $10^{\text {th }}$ International Offshore and Polar Engineering Conference, 94-101, 2000

3. Y. Cao, G. Tachiev, ASME 2013 32nd International Conference on Ocean, Offshore and Arctic Engineering, OMAE2013-11471, (2013)

4. J.W. Kim, H. Jang, J. Kyoung, A. Baquet, J. O'Sullivan, Offshore Technology Conference, 26060 (2015)

5. M.H. Kim, B.J. Koo, R.M. Mercier \& E.G. Ward, Ocean Eng. J. 32, 1780-1802 (2005)

6. Oil Companies International Marine Forum, Prediction of Wind and Current Loads on VLCCs (Witherby \& Co. Ltd., London, 1994) 